Modern diagnostic systems are characterized by that
the flow of diagnostic information requires significant com-
putational resources to process. In order to improve the
reliability of the object to be diagnosed and reduce operat-
ing costs, it is necessary to improve procedures for analyz-
ing diagnostic results. This paper suggests a procedure to
form the diagnostic features of locomotive nodes based on
the use of a principal components analysis. The proposed
approach is distinguished by a decrease in the dimension-
ality of the input set of diagnostic features in order to select
the sets of interconnected diagnostic parameters. Based on
the selection of the sets of interconnected diagnostic fea-
tures, constructing new latent diagnostic parameters has
been proposed. A latent diagnostic parameter contains
information that combines data from several initial diag-
nostic features. The result of the method is a set of latent
diagnostic parameters that do not correlate zith each other
and reflect the behavior of the object to be diagnosed from
different technical points. The application of a sufficient
number of latent diagnostic parameters involved the scree
test method. This paper reports the results from using the
proposed approach for treating the results from diagnos-
ing the hydraulic transmissions in locomotives. The result
from applying the procedure has made it possible to pro-
pose using three latent diagnostic parameters to assess the
technical condition of a locomotive's hydraulic transmis-
sion during bench tests. The suggested parameters contain
90\% of the original information and reflect losses in the
transmission, as well as the load at the input and output of
the transmission
Keyzords: principal components analysis, parameter
informativeness, latent diagnostic parameter, hydraulic
transmission

UDC 629.083

DOI: $10.15587 / 1729-4061.2021 .230293$

\section{DEVISING \\ A PROCEDURE TO \\ FORM THE DIAGNOSTIC \\ PARAMETERS FOR \\ LOCOMOTIVES \\ USING A PRINCIPAL COMPONENTS ANALYSIS}

\author{
Borys Bodnar \\ Doctor of Technical Sciences, Professor, \\ First Vice-Rector* \\ E-mail: bodnar@nz.diit.edu.ua \\ O leks andr Ochkasov \\ PhD, Associate Professor* \\ E-mail: abochkasov@gmail.com \\ *Department of Locomotives \\ Dnipro National University of Railway Transport \\ named after Academician V. Lazaryan \\ Lazaryana str., 2, Dnipro, Ukraine, 49010
}

Received date 24.02.2021 Accepted date 16.04.2021 Published date 20.04.2021
How to Cite: Bodnar, B., Ochkasov, O. (2021). Devising a procedure to form the diagnostic parameters for locomotives using a principal components analysis. Eastern-European Journal of Enterprise Technologies, 2 (1 (110)), $97-103$. doi: https://doi.org/10.15587/1729-4061.2021.230293

\section{Introduction}

The rapid development of information systems has led to a sharp increase in the amount of information that characterizes any sphere of human activity. Vehicle operations and repairs are also accompanied by the generation, processing, analysis, transmission, and preservation of significant amounts of data. One of the areas in the application of big data analysis systems is the analysis of the results from diagnosing vehicles. The development of big data analytics systems is one of the key technologies associated with the fourth industrial revolution. The current direction of the introduction of this technology in engineering is to expand the capabilities of existing and newly created technical diagnostic systems.

The tasks of technical diagnosing include the task of determining the technical condition of an object. The result from solving the problem is the complete or partial removal of uncertainty about the status of the object to be diagnosed. When creating diagnostic systems, an approach can be used in which the maximum possible number of control parameters is measured to monitor the technical condition of vehicles. The use of such an approach is not rational for both economic and technical reasons. Thus, the cost of the diagnostic system increases significantly while some of the control parameters would be redundant.

At the stage of creating the first systems for diagnosing traction rolling stock, the task of selecting a minimum number of diagnostic parameters was relevant. The minimum set of diagnostic parameters is understood to be such a minimum list of controlled parameters, which makes it possible to fully distinguish all possible technical states of the object of control.

At the current stage of technological diagnostics development, the use of data mining technologies is relevant. The purpose of these technologies is to highlight the most informative and generate new diagnostic features. The introduction of intelligent analysis technologies could result in increased vehicle reliability and traffic safety, as well as lower operating and maintenance costs. In addition, the theoretical and practical results of such works would be in demand in the creation and implementation of automated fleet management systems for vehicles.

\section{Literature review and problem statement}

Safety and reliability of operation is a major factor at all stages of the railroad rolling stock service life. An analysis of 
the root causes of serious incidents and disasters is reported in work [1]. The authors single out rolling stock derailment as the main cause of serious incidents and disasters. To improve safety in rolling stock traffic, the authors proposed an improved criterion for assessing the stability of rail carriages against derailment. A study into the reduction of operational costs of transportation companies by decreasing the wear of a wheel-rail pair, as well as by bringing down energy cost of train traction, is reported in [1,2]. One of the fastest-growing areas of research related to increasing the reliability of rolling stock and reducing operating costs is the introduction of individual repair strategies, the introduction of adapted and flexible approaches to the maintenance of locomotives, which are based on the results from technical diagnostics.

The role of technical diagnostics, its impact on the system that maintains and ensures the reliability of traction rolling stock, is described in [3]. The author gives a theoretical justification for the need to introduce technical diagnostics in improving locomotive maintenance systems. The development of diagnostic systems begins with the development of diagnostic models. An overview of the methods for building diagnostic models of traction rolling stock is given in work [4]. The diagnostic models used are the differential equations, logical ratios between parameters, the graphs of cause-and-effect relationships, etc. The process of selecting diagnostic parameters consists of two stages. The first step is to identify a set of parameters describing the state of the object to be diagnosed; the second stage implies compiling, according to certain criteria, such a list of parameters which could provide for the predefined amount of information about the technical condition of the object to be diagnosed.

The main criterion for selecting the set of diagnostic parameters is the information criterion whose application provides for maximum informativeness about the technical condition of the object to be diagnosed.

The information criterion for assessing the informativeness of a diagnostic parameter is proposed in work [5] to build algorithms for the search for faults in radio-electronic equipment. The use of this criterion to build fault-finding algorithms in locomotives' electric circuits is proposed by the authors of paper [6]. The object to be diagnosed is considered as a set of structural units. The technical condition of each structural unit (serviceable/defective) can be determined through elementary verification $\pi_{i}$.

The amount of information one acquires when $\pi_{i}$ is verified is determined from the following formula:

$$
I(\pi)=-\sum P(a) \cdot \log _{2} P(a)-\sum P(b) \cdot \log _{2} P(b),
$$

where $P(a)$ is the probability of verifying $\pi_{i}$ with the outcome $a$ (serviceable); $P(b)$ is the probability of verifying $\pi_{i}$ with the outcome $b$ (defective).

This criterion is used both to select a list of controlled parameters and to build a fault search algorithm. Paper [7] provides an example of the use of the criterion when constructing a technique for diagnosing the electric locomotive. The application of this criterion does not take into consideration the technical capability to measure a given parameter, the cost of control execution, the severity of the possible consequences from the misdiagnosis.

As an improvement of the approach considered, the information and cost criterion was proposed in work [5].

$$
F\left(\pi_{i}\right)=\frac{I\left(\pi_{i}\right)}{C\left(\pi_{i}\right)}
$$

where $I\left(\pi_{i}\right)$ is the amount of information received when verifying $\pi_{i} ; C\left(\pi_{i}\right)$ is the cost of this $\pi_{i}$ verification, which takes into consideration not only material but also time costs.

This approach takes into consideration not only the amount of information received during the diagnostic $\pi_{i}$ verification but also the cost of diagnosing. The use of information and cost criterion is rational in the construction of stationary diagnostic complexes.

To select a set of diagnostic parameters in the construction of on-board diagnostic systems, work [4] proposed using an information-weight criterion.

$$
G\left(\pi_{i}\right)=\frac{I\left(\pi_{i}\right)}{B\left(\pi_{i}\right)}
$$

where $I\left(\pi_{i}\right)$ is the amount of information one receives when $\pi_{i}$ is verified; $B\left(\pi_{i}\right)$ is the coefficient of the relative weight of failure of an element from the $\mathrm{p}_{i}$ system.

To determine the relative weight $B\left(\pi_{i}\right)$ of the failure of each element $\pi_{i}$ of the diagnosed object, paper [4]:

$$
B\left(\pi_{i}\right)=\frac{T\left(\pi_{i}\right) \cdot\left(C\left(\pi_{i}\right)+S\left(\pi_{i}\right)\right) \cdot \lambda(t)_{i}}{\sum_{1}^{m}\left(C\left(\pi_{i}\right)+S\left(\pi_{i}\right)\right) \cdot \lambda(t)_{i}}
$$

where $m$ is the number of structural units analyzed in OD (object to be diagnosed) model; $\lambda\left(t_{i}\right)$ is the failure rate of the $i$-th structural unit; $C\left(\pi_{i}\right)$ is the amount of loss due to the failure of element $\pi_{i} ; S\left(\pi_{i}\right)$ is the cost of restoring an element $\pi_{i} ; T\left(\pi_{i}\right)$ is the severity factor of the consequences of the $\pi_{i}$ element failure.

Using criteria (2), (3) makes it possible to take into consideration both the technical and economic components. The disadvantage of the methods reviewed is that the object to be diagnosed is considered as a set of structural units, each of which is characterized by only two states: serviceable/defective. Typically, these methods are used to develop systems for diagnosing electric vehicle circuitry.

The task of selecting a set of diagnostic parameters for a locomotive in general and for its nodes and assemblies is relevant as the introduction of technical diagnostics could reduce the cost of locomotive maintenance. The relationship between a locomotive readiness coefficient, performance indicators, and a maintenance system has been confirmed by authors in [8]. Paper [9] addresses the choice of diagnostic parameters for the insulation of traction electric motors in locomotives. The cited paper explores the technical condition of insulation using non-destructive control methods. The results of a study on assessing the technical condition of the mechanical equipment of electric locomotives are reported in [10].

At the current stage of development of the traction rolling stock, technical diagnostic systems are generally integrated with automated control systems. Even though the technical condition of most locomotive nodes is monitored, this approach has a series of significant drawbacks. Namely: a significant number of sensors and control devices, large volumes of diagnostic information, high cost of the technical complex.

The processing of information in diagnostic systems is based on the formalization of the original attributes and the identification of space of valuable features. The value of a diagnostic feature is considered in terms of maximizing 
information about the technical condition of the object to be diagnosed. The methods of choosing diagnostic features include mathematical procedures for assessing the informativeness of diagnostic features. Traditional procedures are used that employ variance, regression, correlational analyses, as well as a theoretical-information approach based on the calculation of the probabilities and the amount of information, and multidimensional statistical analysis. Paper [11] examines the use of statistical methods and a probabilistic approach to predicting the failure of railroad automation systems.

In the development of diagnostic support for complex systems, construction of diagnostic models, categorization, and forecasting of the condition, a series of mathematical problems arise. Namely, the large dimensionality of diagnostic data; values of certain control parameters correlate with each other; low informativeness of some control parameters.

The locomotive information system measures so many parameters, the volume and speed of which is much greater than a person's ability to conduct analysis. During the operation of the locomotive, a data stream is generated, in which it is necessary to highlight the information components of the changing technical condition of the object to be diagnosed. Our review of studies [5-11] suggests that, despite a significant body of research in this area, the task of selecting the most informative diagnostic features continues to be relevant. The focus of further research development would be to select the most informative components in order to automate and accelerate the interpretation of diagnostic results.

In the development of vehicle diagnostic support, authors propose assessing the informativeness of diagnostic parameters by using mathematical methods to reduce dimensionality. They focus, among methods to reduce dimensionality, on a principal components analysis, a factor analysis, the extreme parameter grouping, the choice of the most informative features in the discriminate model analysis, and regression models, etc.

The main theoretical approaches to the intelligent analysis of statistics using a principal components analysis are outlined in papers [12-14].

The authors of [15] used a principal components analysis and a hierarchy analysis method to build an index of an arbitrary process, which allowed them to select the most informative statistical parameters and assess the quality of the process under study.

Papers [16, 17] provide an overview of the approaches to the use of a principal components analysis and its development when used in science and technology.

Examples of applying a principal components analysis to estimate the state of traffic safety, as well as the operational indicators of a locomotive fleet, are given in works [18, 19].

The application of a principal components analysis for diagnosing malfunctions in equipment is reported in [20-23]. The results of those works allow us to assert the effectiveness of using the method to detect faults in complex technical objects.

\section{The aim and objectives of the study}

The aim of this study is to devise a procedure to reduce the dimensionality of the set of diagnostic parameters while preserving the maximum information about the technical condition of the object to be diagnosed. That would make it possible to improve the accuracy in determining the technical condition of the object to be diagnosed without increas- ing the number of control parameters, to reduce the time and resource costs on interpreting the results from diagnosing. Achieving the goal could reduce operating costs by improving the reliability of the object to be diagnosed.

To accomplish the aim, the following tasks have been set:

- to suggest a way to reduce the dimensionality of the set of diagnostic parameters;

- to propose a technique to select the number of diagnostic parameters enough to maintain the predefined amount of information about the status of the object to be diagnosed;

- to construct a set of latent diagnostic parameters, so that the elements of the set do not correlate with each other.

\section{Materials and methods to form the diagnostic features of locomotives using a principal components analysis}

Based on the above analysis, we have selected a principal components analysis as a mathematical apparatus that makes it possible to analyze available diagnostic features. The main purpose of this method is to obtain a set of new latent diagnostic parameters that provide for maximum informativeness. At any given time, the technical condition of the object to be diagnosed can be represented by the vector $X=\left\{x_{1}, x_{2}, x_{3} \ldots x_{n}\right\}$ of dimensionality $n$; as a result of the use of a principal components analysis, the original vector is converted into the vector $G=\left\{g_{1}, g_{2}, g_{3} \ldots g_{n}\right\}$. In further analysis, the dimensionality of the principal components' vector is reduced to $m$ (where $m \leq n$ ) most informative components.

Thus, the original $n$-dimensional space of diagnostic parameters is reduced to an $m$-dimensional space of information components, each of which contains aggregated information about the technical condition of the object to be diagnosed. From a technical point of view, the $g_{i}$ component is the new diagnostic parameter that characterizes the object to be diagnosed. The $G$ component set contains a set of diagnostic parameters, each containing maximum information about one of the technical aspects of OD, in this case, the parameters are minimally related. The set of $G$ components can be used for further analysis to determine the technical condition, for diagnostics, and estimating the residual resource.

Mathematically, converting the original set of diagnostic parameters into a set of diagnostic components is carried out by the linear transformations of inputs to the new normalized features. The new set of diagnostic features contains, in this case, a set of normalized and non-correlated components.

Let us designate a set of original diagnostic features through $X=\left\{x_{n}\right\}_{n=1}^{N}, x_{n} \in R^{D}$. The goal of reducing dimensionality is to obtain, based on the original set of diagnostic parameters, a new set of diagnostic components $G=\left\{g_{n}\right\}_{n=1}^{N}, g_{n} \in R^{d}$, where $d \ll D$. The original set of features $X=\left\{x_{n}\right\}_{n=1}^{N}$ is the $N$-dimensional «point cloud». Using factor analysis methods, it is required to find planes passing through the center of gravity of the «cloud» so that the sum of the squares of distances from all points to these planes is minimal. When using a principal components analysis, the planes are selected from the condition of maximizing variance in one direction. To obtain the set of diagnostic components, it is necessary to:

- normalize initial data;

- find the correlation matrix $r$;

- find the eigen numbers and eigenvectors of matrix $r$;

- order the eigenvectors and eigen numbers from maximum to minimum. 
The process of obtaining the set of diagnostic components $G$ is iterative. The first step would determine the plane relative to which the variance of the original diagnostic parameters is maximum. The next steps select the following planes, relative to which the variance of the remaining parameters is maximum, while the resulting diagnostic components do not correlate with each other. Upon solving the problem, the number of components to be received would equal the original number of diagnostic features. However, there is no point in using all $N$ components. The choice of a sufficient number of diagnostic components is based on the scree test criterion [24]. The proposed criterion is analyzed graphically. The essence of using the scree test criterion is to determine the number of a component for which the decline in the factors' eigenvalues slows down in the most intensive way.

The result of using the scree test criterion is the derived $M$-dimensional set of diagnostic components $G$, each of which contains the maximum information about the condition of the object and characterizes different aspects of the object to be diagnosed.

The original diagnostic component includes $N$ parameters:

$$
g_{i}=\sum_{i=1}^{N} x_{i} \cdot a_{i}
$$

where $x_{i}$ is the value of a diagnostic parameter; $a_{i}$ is the factor load on a parameter; $N$ is the dimensionality of the original set of diagnostic parameters.

Significant $M$ diagnostic components are determined from formula (6):

$$
g_{i}=\sum_{i=1}^{M} x_{i} \cdot a_{i}
$$

where $M$ is the number of significant components defined from the scree test.

The contribution (informativeness) of the $x_{i}$ parameter is greater, the greater the absolute value of $\left|a_{i}\right|$. The $a_{i}$ factor load sign indicates the direction of change in the original diagnostic parameter $x_{i}$ relative to the main component axis. The number of initial diagnostic parameters $x_{i}$ that are part of each component is determined based on the factor load values of the parameters and the physical sense of the parameters.

\section{Results of the formation of diagnostic parameters for locomotive nodes}

\footnotetext{
5. 1. Reducing the dimensionality of the set of diagnostic parameters

Bench tests of hydraulic transmissions such as UGP750/12000 have been selected as the object of research using the proposed approach to form the diagnostic features of locomotive nodes. After a major repair, hydraulic transmission is tested at a specialized bench; a series of control parameters are measured during the tests. The specifications of the testing equipment, the procedure of treating and analyzing the results are given in papers [25-27].
}

The results from the bench tests of 10 hydraulic transmissions have been analyzed. The following diagnostic parameters were registered during the tests: the voltage $U_{m}$ and the armature current $I_{m}$ of the drive electric motor; the voltage $U_{\text {gen }}$ and the armature current $I_{g e n}$ of the load electric motor. For a hydraulic transmission, the input parameters are the rotation frequency $w_{m}$ and the torque $M_{m}$ of the drive electric motor; the rotation frequency $w_{c i}$ and the torque of the pumping wheel $M_{c i}$. The output parameters of the hydraulic transmission during tests are the rotation frequency $w_{c t}$ and the torque of the turbine wheel $M_{c t}$, which corresponds to the rotation frequency and the torque of the load generator armature. The temperature of the oil at the input $t_{i n p}$ and output $t_{\text {out }}$ of the hydraulic transmission, the temperature $t_{t c f}$ and pressure of oil $p_{t c f}$ in the hydraulic apparatus are also registered. In addition, the estimation parameters were taken into consideration. The power of the drive electric motor $P_{m}$ and the power of the load generator $P_{\text {gen }}$. The ratio of the oil temperature in the hydraulic apparatus to the power of the generator $t_{t c f} / P_{\text {gen }}$. The transmission ratio of rotational frequencies of the turbine $w_{c t}$ and pumping wheels $w_{c i}$.

The results of treating the data set on the hydraulic transmission tests using the proposed procedure are given in Tables 1, 2. Examples include calculations for one hydraulic transmission.

Table 1 gives the amount of information for each component. When using the scree test criterion, 3 components $\left\{g_{1}, g_{2}, g_{3}\right\}$ would suffice to describe the technical condition of the hydraulic transmission on the bench. In total, these three components contain $90 \%$ of the original information.

Table 2 gives the values of factor loads of diagnostic parameters $a_{1}, \ldots, a_{14}$ for the first three components.

The calculated factor loads of diagnostic parameters are used to form latent diagnostic parameters. When selecting the number of diagnostic parameters that make up the component, we consider the value of the factor load taken by the module. Select the parameters that have the greatest factor load in each component. For example, for component 1, we choose generator voltage, generator current, pressure in the hydraulic apparatus.

5. 2. Choosing the number of diagnostic parameters enough to maintain the predefined amount of information

The results of our calculation of the components' informativeness for one hydraulic transmission the type of UGP 750/1200 are shown in Fig. 1. Based on the calculation results (Fig. 1 and Table 1), we can conclude that the technical condition of the hydraulic transmission in bench tests can be described with the help of three components. The amount of information contained in the first three components of other hydraulic transmissions of the same type is in the range of 86 to $92 \%$.

Determining the number of components enough to describe a technical condition implies taking into consideration the possibility of interpreting the technical aspects reflected by the components. In a given example, three components are accepted. If only the first two components are used, $82 \%$ of the original information would be available for further analysis.

The informativeness of components $G=\left\{g_{1} \ldots g_{14}\right\}$

Table 1

The informativeness of components $G=\left\{g_{1} \ldots g_{14}\right\}$

\begin{tabular}{|c|c|c|c|c|c|c|c|c|c|c|c|c|c|c|}
\hline Component number & 1 & 2 & 3 & 4 & 5 & 6 & 7 & 8 & 9 & 10 & 11 & 12 & 13 & 14 \\
\hline Informativeness, \% & 54.6 & 27.6 & 7.9 & 3.3 & 2.0 & 1.5 & 1.2 & 0.9 & 0.3 & 0.3 & 0.2 & 0.1 & 0.1 & 0.1 \\
\hline
\end{tabular}


Table 2

Diagnostic parameters factor loads

\begin{tabular}{|c|c|c|c|}
\hline Parameter & Component 1 & Component 2 & Component 3 \\
\hline Drive motor voltage, $U_{m}$ & -0.0745 & -0.0068 & -0.4637 \\
\hline Drive engine current, $I_{m}$ & 0.0467 & 0.2385 & -0.4752 \\
\hline Generator voltage, $U_{\text {gen }}$ & 0.3519 & 0.1553 & -0.0449 \\
\hline Generator current, $I_{\text {gen }}$ & -0.8122 & 0.1895 & 0.0621 \\
\hline Drive engine rotation frequency, $w_{m}$ & -0.0092 & 0.0069 & -0.2026 \\
\hline Pump wheel rotation frequency, $w_{c i}$ & 0.0065 & 0.0060 & -0.0202 \\
\hline Turbine wheel rotation frequency, $w_{c t}$ & 0.0499 & 0.0024 & -0.0758 \\
\hline Oil temperature in hydraulic apparatus, $t_{t c f}$ & 0.2238 & 0.512 & 0.2016 \\
\hline Oil pressure in hydraulic apparatus, $p_{t c f}$ & -0.3281 & 0.1709 & -0.3606 \\
\hline Oil temperature at the transmission input, $t_{i n p}$ & 0.0943 & 0.5504 & 0.0263 \\
\hline Oil temperature at the transmission output, $t_{\text {out }}$ & 0.0280 & 0.5288 & 0.2399 \\
\hline Generator power $P_{\text {gen }}$ & 0.1297 & -0.0622 & -0.0072 \\
\hline Drive motor power, $P_{m}$ & -0.0053 & -0.0085 & -0.0844 \\
\hline The ratio of oil temperature to pressure in the hydraulic apparatus, $t_{t c f} / P_{g e n}$ & 0.1476 & -0.0018 & 0.2662 \\
\hline The ratio of the turbine wheel speed to the pump wheel speed $w_{c t} / w_{c i}$ & -0.0057 & 0.0213 & 0.0081 \\
\hline
\end{tabular}

Determining the number of components enough to monitor the technical condition of a hydraulic transmission

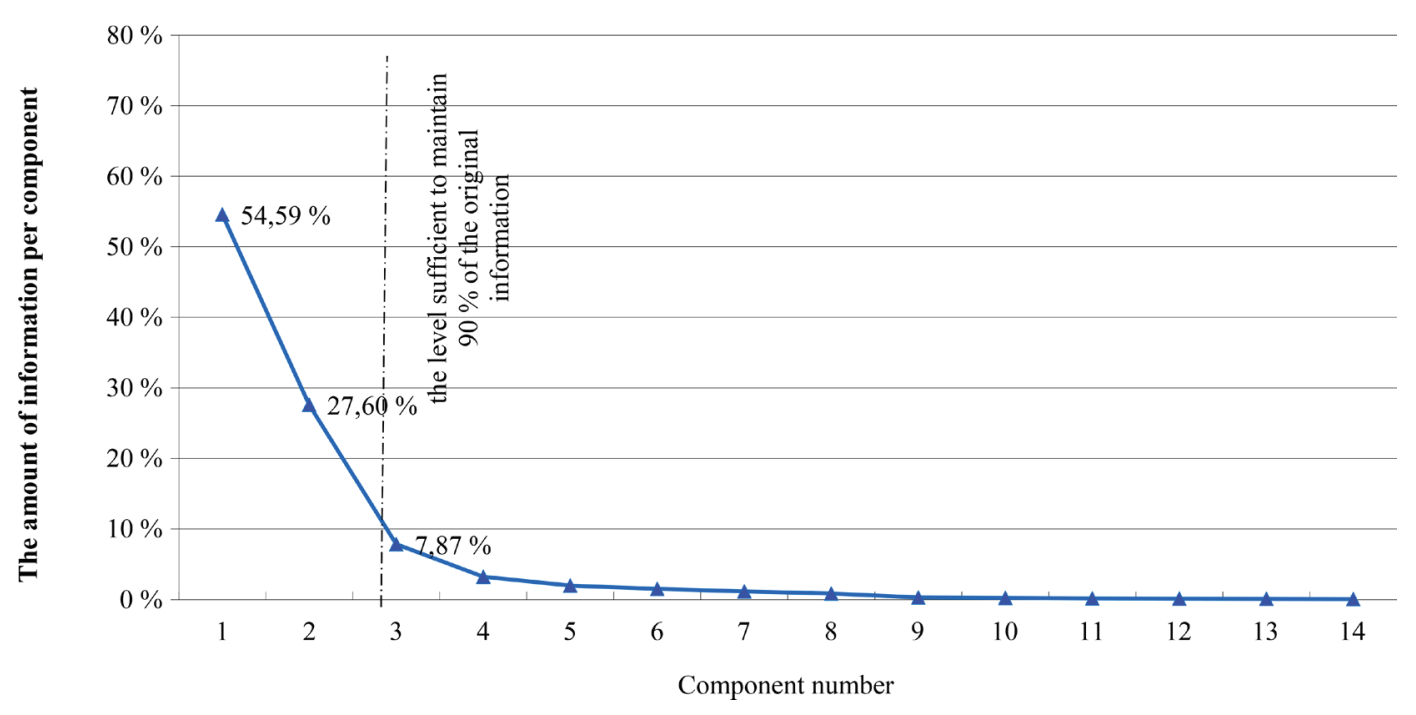

Fig. 1. Determining the number of components enough to monitor the technical condition of a hydraulic transmission during bench tests

\section{3. Constructing a set of non-correlated latent diag-} nostic parameters

The factor loads of the tested hydraulic transmissions are distributed similarly to Table 2 . The greatest factor load in the first component is on the following parameters: the voltage $U_{\text {gen }}$ and the armature current $I_{\text {gen }}$ of the loading electric motor; oil pressure in the hydraulic apparatus $p_{t c f}$. The diagnostic parameters that make up the first component characterize the load on a hydraulic transmission during the testing process. Thus, the first component «load» characterizes the response of the hydraulic transmission to the load during the tests. The second component includes such parameters as the temperature of oil at the input $t_{\text {inp }}$ and output $t_{\text {out }}$ of the hydraulic transmission; the temperature of oil in the hydraulic apparatus $t_{t c f}$. These three quantities characterize the value of hydraulic transmission loss during tests. The second component can be conventionally called «loss»; it indirectly characterizes the efficiency of transmission. The third component «input» characterizes the power of the drive engine; it includes the voltage $U_{m}$ and the armature current $I_{m}$ of the drive electric motor.

Thus, the application of a principal components analysis has produced three latent diagnostic features: load, loss, input. The values of these features can be determined using (5). For the hydraulic transmission in question, using an example of Table 2, the component equations take the following form:

$$
\begin{aligned}
& \text { load }=0.3519 \cdot U_{\text {gen }}-0.8122 \cdot I_{\text {gen }}-0.3281 \cdot p_{\text {tcf }}, \\
& \text { losses }=0.512 \cdot t_{t c f}-0.5504 \cdot t_{\text {inp }}-0.5288 \cdot t_{\text {out }}, \\
& \text { input }=-0.4637 \cdot U_{m}-0.4752 \cdot I_{m} .
\end{aligned}
$$

The components' numerical values are dimensionless quantities. Using the values of components and factor loads, the technical condition of hydraulic transmissions is compared and evaluated during the bench tests. 


\section{Discussion of results of forming the diagnostic parameters using a principal components analysis}

The key point of our study is the use of a principal components analysis as one of the methods to reduce dimensionality, to highlight the most informative components from the original set of diagnostic parameters. The concept of a «latent diagnostic parameter» has been introduced in the technical diagnostics of locomotive nodes. The process of forming latent diagnostic parameters involves the following basic stages:

1. Convert the original set of diagnostic parameters $X=\left\{x_{1}, x_{2}, x_{3}, \ldots, x_{n}\right\}$ into a set of diagnostic components $G=\left\{g_{1}, g_{2}, g_{3}, \ldots, g_{n}\right\}$. The conversion implies using a principal components analysis.

2. Based on the calculated factor loads, we derive a set of linear equations for each principal component, represented by expression (6).

3 . The scree test method is used to determine the sufficient number of diagnostic components. Employing this approach reduces the dimensionality of the set of diagnostic parameters while maintaining the predefined amount of information about the condition of the object. An example of using the method is shown in Fig. 1. The equations of latent diagnostic parameters are described by expressions (6).

A special feature of the proposed approach, unlike other methods, is that the resulting latent diagnostic parameters do not correlate with each other. Thus, they make it possible to characterize the object to be diagnosed from different technical sides. Tables 1, 2 give the results of the calculation, which produced such latent diagnostic parameters as load, losses, and input.

The main limitation of the use of a principal components analysis and the proposed approach to forming latent diagnostic parameters is the approximation of the original set of parameters by straight lines and planes. If the original set of diagnostic features is better approximated by non-linear dependences, the effectiveness of the proposed approach is reduced. In this case, it is rational to use the principal manifold method or a nonlinear principal component method.

The issue of determining the number of latent diagnostic parameters (components) enough to preserve the predefined amount of information is debatable. Fig. 1 shows a rationale for choosing the number of components when diagnosing a hydraulic transmission in a locomotive. The scree test has been used to determine the number of latent diagnostic parameters. Based on the experience of testing hydraulic transmissions of this type, the application of three latent diagnostic parameters makes it possible to retain from 86 to $92 \%$ of the original information. As the percentage of the original information to be saved increases, the number of latent parameters rises. In this case, it is possible to include some non-informative components that could complicate the interpretation of the results. Reducing the number of latent diagnostic parameters would lead to a decrease in the accuracy of diagnosing. The final decision on the specified level of keeping the original data can be made on the basis of analysis of the components' factor loads and technical interpretation of the results.

As a continuation of this study, it is planned to apply the proposed approach to analyze the diagnostic information about the locomotive in general. And when designing automated expert systems to categorize the technical conditions of objects to be diagnosed. The resulting component values can be used for further processing using clustering methods, regression analysis to predict changes in technical condition and determine the residual resource.

Further research aims to investigate the relationship between the factor loads of the parameters included in components and the degree of informativeness of diagnostic components and a hydraulic transmission technical condition. The proposed technique could be used in the analysis of the test results and for diagnosing other locomotive nodes.

\section{Conclusions}

1. Based on the application of a principal components analysis, a technique to reduce the dimensionality of the set of diagnostic parameters has been proposed. Using a principal components analysis has made it possible to distinguish 8 most informative diagnostic parameters from the original set of 14 parameters.

2. The original diagnostic parameters were combined on the basis of the values of factor loads of parameters in each principal component. The application of a scree test has enabled the selection of a sufficient number of diagnostic components. Three diagnostic components are recommended to preserve information about the technical condition of a hydraulic transmission during bench tests.

3. We have introduced the concept of a latent diagnostic parameter for a hydraulic transmission - a parameter derived from combining several interconnected quantities. The special feature of latent diagnostic parameters is that the parameters are minimally correlated with each other and, at the same time, make it possible to assess the condition of the object to be diagnosed from different technical perspectives. Based on factor load values, three latent diagnostic parameters «loss,» «load», and «input» have been formed. We determined the values of the latent diagnostic parameters using the 8 most informative diagnostic parameters; the resulting latent parameters contain $90 \%$ of the original information. The largest amount of information about the technical condition of a hydraulic transmission during bench tests is contained in the «load» parameter $(54.6 \%)$, the «loss» parameter contains $27.6 \%$ of the information, the «input» parameter accounts for $7.87 \%$ of the original information.

\section{References}

1. Tkachenko, V., Sapronova, S., Kulbovskiy, I., Fomin, O. (2017). Research into resistance to the motion of railroad undercarriages related to directing the wheelsets by a rail track. Eastern-European Journal of Enterprise Technologies, 5 (7 (89)), 65-72. doi: https://doi.org/10.15587/1729-4061.2017.109791

2. Sapronova, S., Tkachenko, V., Fomin, O., Hatchenko, V., Maliuk, S. (2017). Research on the safety factor against derailment of railway vehicless. Eastern-European Journal of Enterprise Technologies, 6 (7 (90)), 19-25. doi: https://doi.org/10.15587/ 1729-4061.2017.116194 
3. Kapitsa, M. I., Laguta, V. V. (2013). Modeli rezhimov diagnostirovaniya tyagovogo podvizhnogo sostava s zamenoy komplektuyuschih izdeliy. Elektromahnitna sumisnist ta bezpeka na zaliznychnomu transport, 5, 56-62.

4. Bodnar', B. E., Ochkasov, A. B. (2001). Vybor diagnosticheskih parametrov s ispol'zovaniem informatsionno-vesovogo kriteriya. Sbornik trudov DIIT: Transport, 7, 35-37.

5. Pashkovskiy, G. S. (1981). Zadachi optimal'nogo obnaruzheniya i poiska otkazov v REA. Moscow: Radio i svyaz', 280.

6. Pushkarev, I. F., Strekopytov, V. V. (1988). Nadezhnost' i tehnicheskaya diagnostika lokomotivov. Leningrad, 61.

7. Lin, L., Jiang, X., Huang, Z., Hu, H. (2010). Application of advanced fault diagnosis technology in electric locomotives. International Journal of Modelling, Identification and Control, 10 (3/4), 292. doi: https://doi.org/10.1504/ijmic.2010.034581

8. Falendysh, A., Sumtsov, A., Artemenko, O., Klecka, O. (2016). Simulation of changes in the steady state availability factor of shunting locomotives for various maintenance systems. Eastern-European Journal of Enterprise Technologies, 1 (3 (79)), 24-31. doi: https://doi.org/10.15587/1729-4061.2016.60640

9. Kapitsa, M., Laguta, V., Kozik, Y. (2018). Selecting the Parameters of The Diagnosis of Frame Insulation Condition in Electrical Machines of Locomotives. International Journal of Engineering \& Technology, 7 (4.3), 110. doi: https://doi.org/10.14419/ijet.v7i4.3.19718

10. Bannikov, D., Yakovlev, S. (2020). Development of dynamic integral evaluation method of technical state of one-section electric locomotive body. Eastern-European Journal of Enterprise Technologies, 1 (7 (103)), 57-64. doi: https://doi.org/10.15587/ 1729-4061.2020.192468

11. Moiseenko, V., Kameniev, O., Gaievskyi, V. (2017). Predicting a technical condition of railway automation hardware under conditions of limited statistical data. Eastern-European Journal of Enterprise Technologies, 3 (9 (87)), 26-35. doi: https:// doi.org/10.15587/1729-4061.2017.102005

12. Orlov, A. I., Lutsenko, E. V. (2016). Methods of reducing space dimension of statistical data. Nauchniy zhurnal KubGAU, 119, 92-107. Available at: https://elibrary.ru/item.asp?id $=26148522$

13. Ayvazyan, S. A., Buhshtaber, V. M., Enyukov, I. S., Meshalkin, L. D.; Ayvazyan, S. A. (Ed.) (1989). Prikladnaya statistika: Klassifikatsii i snizhenie razmernosti. Moscow: Finansy i statistika, 607.

14. Subbotin, S. A. (2013). Sample formation and reduction for data mining. Radio Electronics, Computer Science, Control, 1, 113-118 doi: https://doi.org/10.15588/1607-3274-2013-1-18

15. Bosov, A., Loza, P. (2014). Creation of an index of arbitrary process. Zbirnyk naukovykh prats Donetskoho instytutu zaliznychnoho transportu, 38, 68-73. Available at: http://nbuv.gov.ua/UJRN/znpdizt_2014_38_13

16. Yin, S., Ding, S. X., Xie, X., Luo, H. (2014). A Review on Basic Data-Driven Approaches for Industrial Process Monitoring. IEEE Transactions on Industrial Electronics, 61 (11), 6418-6428. doi: https://doi.org/10.1109/tie.2014.2301773

17. Jolliffe, I. T., Cadima, J. (2016). Principal component analysis: a review and recent developments. Philosophical Transactions of the Royal Society A: Mathematical, Physical and Engineering Sciences, 374 (2065), 20150202. doi: https://doi.org/10.1098/ rsta.2015.0202

18. Bodnar, B., Bolzhelarskyi, Y., Ochkasov, O., Hryshechkina, T., Černiauskaite, L. (2018). Determination of integrated indicator for analysis of the traffic safety condition for traction rolling stock. Paper presented at the 12th International Conference on Intelligent Technologies in Logistics and Mechatronics Systems, ITELMS 2018. Panevėžys, 45-54.

19. Bodnar, B., Ochkasov, O., Bodnar, E., Hryshechkina, T., Keršys, R. (2018). Safety performance analysis of the movement and operation of locomotives. Proceedings of 22nd International Scientific Conference, 839-843.

20. Nadir, F., Elias, H., Messaoud, B. (2020). Diagnosis of defects by principal component analysis of a gas turbine. SN Applied Sciences, 2 (5). doi: https://doi.org/10.1007/s42452-020-2796-y

21. Mnassri, B., Adel, E. M. E., Ananou, B., Ouladsine, M. (2009). Fault Detection and Diagnosis Based on PCA and a New Contribution Plot. IFAC Proceedings Volumes, 42 (8), 834-839. doi: https://doi.org/10.3182/20090630-4-es-2003.00137

22. Doorsamy, W., Cronje, W. A. (2015). A method for fault detection on synchronous generators using modified principal component analysis. 2015 IEEE International Conference on Industrial Technology (ICIT). doi: https://doi.org/10.1109/icit.2015.7125162

23. Zheng, M., Wu, L., Li, L., Liu, C., Wang, L., Sun, S. (2017). A modified method for fault detection and isolation of redundant inerial measurement unit in dynamic environment. 2017 36th Chinese Control Conference (CCC). doi: https://doi.org/10.23919/ chicc.2017.8028460

24. Cattell, R. B. (1966). The Scree Test For The Number Of Factors. Multivariate Behavioral Research, 1 (2), 245-276. doi: https:// doi.org/10.1207/s15327906mbr0102_10

25. Zhukovytskyy, I. V., Kliushnyk, I. A., Ochkasov, O. B., Korenyuk, R. O. (2015). Information-measuring test system of diesel locomotive hydraulic transmissions. Science and Transport Progress. Bulletin of Dnipropetrovsk National University of Railway Transport, 5 (59), 53-65. doi: https://doi.org/10.15802/stp2015/53159

26. Bodnar, B., Ochkasov, O., Bobyr, D., Korenyuk, R., Bazaras, Z. (2018). Using the Self-Braking Method when the Post-Overhaul Diagnostics of Diesel-Hydraulic Locomotives. In: 2018 Transport means proceedings of the international conference. Kaunas, 914-919.

27. hukovyts'kyy, I., Kliushnyk, I. (2018). Development of a selfdiagnostics subsystem of the information-measuring system using anfis controllers. Eastern-European Journal of Enterprise Technologies, 1 (9 (91)), 11-19. doi: https://doi.org/10.15587/17294061.2018.123591 